\title{
Sparse Eigenmotions Derived from Daily Life Kinematics Implemented on a Dextrous Robotic Hand
}

\author{
Charalambos Konnaris ${ }^{1}$, Andreas A. C. Thomik ${ }^{1}$ and A. Aldo Faisal ${ }^{1,2,3}$, Member IEEE
}

\begin{abstract}
Our hands are considered one of the most complex to control actuated systems, thus, emulating the manipulative skills of real hands is still an open challenge even in anthropomorphic robotic hand. While the action of the 4 long fingers and simple grasp motions through opposable thumbs have been successfully implemented in robotic designs, complex in-hand manipulation of objects was difficult to achieve. We take an approach grounded in data-driven extraction of control primitives from natural human behaviour to develop novel ways to understand the dexterity of hands. We collected hand kinematics datasets from natural, unconstrained human behaviour of daily life in 8 healthy in a studio flat environment. We then applied our Sparse Motion Decomposition approach to extract spatio-temporally localised modes of hand motion that are both time-scale and amplitude-scale invariant. These Sparse EigenMotions (SEMs)[1] form a sparse symbolic code that encodes continuous hand motions. We mechanically implemented the common SEMs on our novel dexterous robotic hand [2] in open-loop control. We report that without processing any feedback during grasp control, several of the SEMs resulted in stable grasps of different daily life objects. The finding that SEMs extracted from daily life implement stable grasps in openloop control of dexterous hands, lends further support for our hypothesis the brain controls the hand using sparse control strategies.
\end{abstract}

\section{INTRODUCTION}

Our hands are considered to be highly dexterous endeffectors comprised by various types of joints. They serve as precision tools and allow us to interact with the world with little cognitive effort. This seamless operation of our hands, masks the complex biomechanical and neural control strategies deployed by the brain to orchestrate the movements of our hands 24+ degrees of freedom (DoF)[3]. The Central Nervous System, handles the complexity and high dimensionality of control with amazing ease and absence of effort [3]. Therefore, the search for a low-dimensional, easily interpretable description of motor outputs, has attracted a considerable amount of scientific interest over the past two decades [1]. As a result, hand muscle synergies have been studied extensively [4], [5], in order to investigate the dimensionality of hand control. Muscle synergies describe synchronous co-activation of muscles, time shifted co-activation or more complex time-varying activation patterns of muscles [6]. Although this method has been successful at capturing the observed muscle activations, the validity of these models is still widely debated [7]. Several approaches have been

Brain \& Behaviour Lab - ${ }^{1}$ Dept. of Bioengineering \& ${ }^{2}$ Dept. of Computing, Imperial College London, South Kensington Campus, SW7 2AZ, London, UK, ${ }^{3}$ MRC Clinical Sciences Centre. Address for correspondence: aldo.faisal@imperial.ac.uk taken towards exploiting a lower dimensional manifold to control artificial hands [8], [9].

To understand the principles underlying human hand motor control and reverse engineer these strategies for prosthetics and robotic control of artificial hands we pursue an approach based on studying natural human behaviour. Much of the focus of hand control is on grasping [4], [5], [9], yet hand control involves also more complex behaviours such as inhand object manipulation (e.g. using chop sticks), compound grasps (e.g. using a pen and an eraser held in one hand) and seamless blending between various hand actions [10]. Thus dexterous robotics and prosthetic control function is analysed by constrained experimental protocols, in a way that may not be relevant to how the brain is organised to control hand movements. Instead of using highly controlled stereotyped examples of human hand movements (e.g. repeated grasp of specific objects) we studied hand behaviour in a natural setting [1], [11], a studio flat environment in which subjects lived and worked day long and whose hand kinematics were collected using wearable sensors.

The brain needs to translate sensory inputs into control commands to implement feedback control. In the case of controlling the hand, we assume an efficient coding hypothesis for the motor system [12], .i.e. that the brain's encoding of hand postures should be related to control of hand posture. Specifically, we assume that the representation of hands posture, i.e. the proprioceptive information about hand dynamics are shaped by the natural statistics of hand postures in daily life and geared towards supporting the control of natural hand movements. Many different encodings are possible, each optimal for a specific criterion. While it is unclear what criteria of optimality the brain uses, it has been suggested that due to the significantly larger number of cortical neurons being driven by a comparatively smaller number of sensory neurons [13] that sparse coding may be a good candidate - this was extensively demonstrated in the visual system. In analogy, we apply the concepts to motor control, where the vast number of cortical neurons in primary motor cortex vastly outnumber the number of motor neurons in the spinal cord and their innervated muscles.

We build our work on sparse coding [1] and translate these data-driven models into mechanical implementations on a novel hand [2]. We present a novel approach towards the naturalistic control of dexterous robotic and prosthetic hands by projecting natural motor behaviour in an artificial hand. Using the novel concept of Sparse Coding, we analyse unconstrained human behaviour and subsequently extract the Sparse Eigenmotions (SEMs) of the hand. Finally, we 
implement 4 SEMs on a dexterous artificial hand in order to test our frame-work by exploiting a lower dimensional manifold. Our preliminary results, motivate the creation of a platform that is able to operate a wide range of dexterous robotic and prosthetic hands.

\section{Methods}

\section{A. Data Collection of natural daily life kinematics}

Human sensorimotor control has been predominantly studied using fixed tasks performed in laboratory conditions, neglecting natural hand movements. This method of studying human motor behaviour greatly advanced our understanding of the underlying mechanisms that integrate sensory information and generate motor commands during voluntary tasks. However, kinematic data collected "in-the-wild" has been shown to produce indeed systematic differences to experimentally predefined lab tasks in the same subjects [14], [15]. Therefore, collecting natural behaviour data will allow decoding the underlying motor strategies deployed by the brain in order to control and coordinate movement in unconstrained environments.

We move away from laboratory conditions and we quantify human behaviour in natural unconstrained environment. By incorporating a combination of wireless motion capturing devices, human behaviour is captured in high resolution. We have collected full body kinematic data using Cybergloves (CyberGlove III \& II :22 DoF \& 18 DoF, Cyberglove Inc, USA) on both hands and a motion capturing suit (51 DoF: IGS-180 Animazoo Ltd. , UK). A distinctive attribute of this method of collecting human behaviour data in "the wild", is the ability to overcome limitations of traditional optical markers such as sensor occlusion [16]. Data collection took place in three scenarios: office, kitchen and bedroom. Each scenario lasted about 45 minutes and a total of 8 healthy subjects took part in this experimental study.

\section{B. Sparse Eigenmotions (SEMs) from daily-life activity}

Numerous methods in literature analyse hand kinematic data to their disposal, using Principal Component Analysis (PCA) in order to find an easily interpretable description of motor outputs. We analyse the data collected, using the concept of sparse coding and dictionary learning. This particular method of analysis has proven to provide exceptional encoding of natural images similar to the one found in visual cortex [17]. Sparse coding is a deterministic, iterative procedure that seeks an optimal dictionary of Eigenmotions to represent the input data by computing a short-time PCA on strategically chosen Regions of Interest (ROI) amongst the data-set. The sparse Eigenmotions (SEMs) are then extracted and added to the dictionary (see [1] for details).

We derived a sparse coding representation of human body kinematics and applied this to the analysis of natural kinematics in daily life collected in a studio flat from 8 subjects. Each subject's individual kinematics were analysed and SEMs extracted. SEMs were compared across subjects and conserved SEMs were used for further processing. Our results [1] suggested that sparse coding of hand movements in the brain can be captured by a finite dictionary of primitive movements which are conserved across multiple subjects. Such sparse motions could be combined to create the observed motor output [18] and are consistent with previous studies which found neurons coding specifically

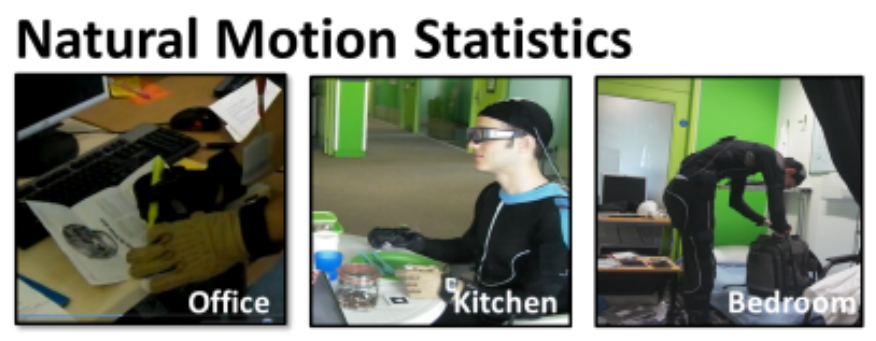

\section{Sparse Eigenmotion Extraction}

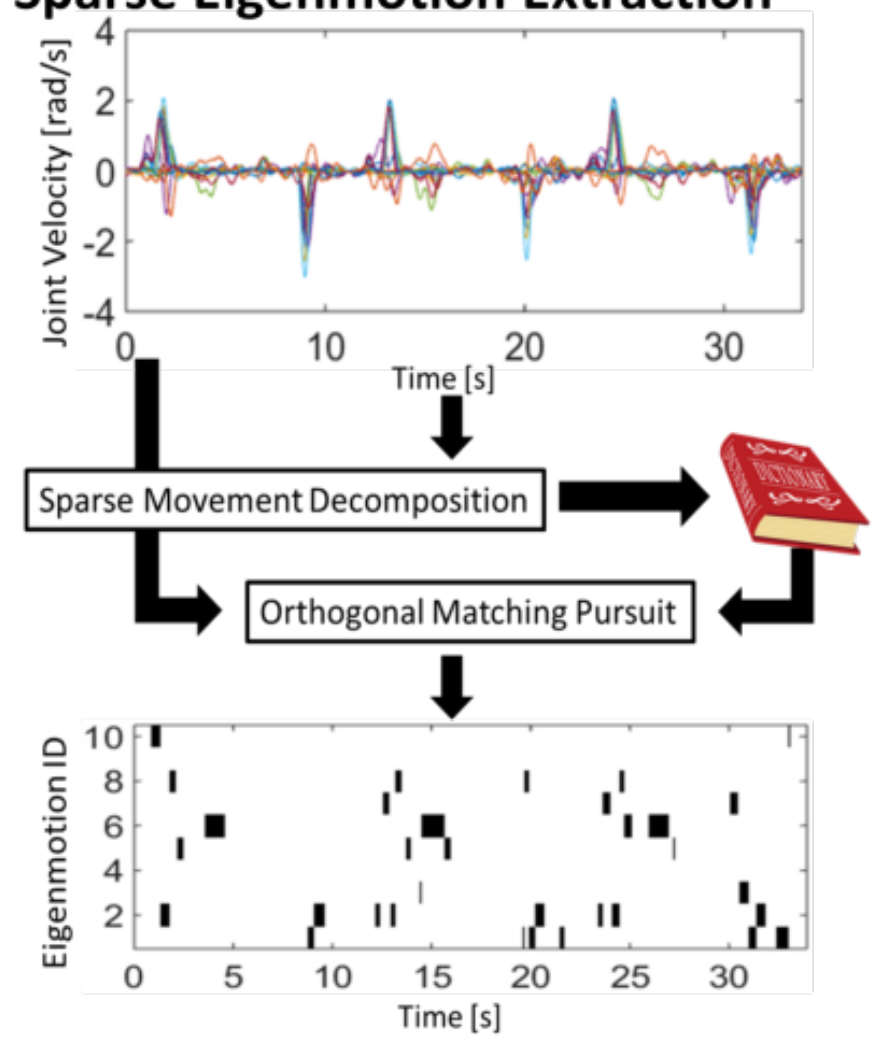

\section{Hardware implementation}

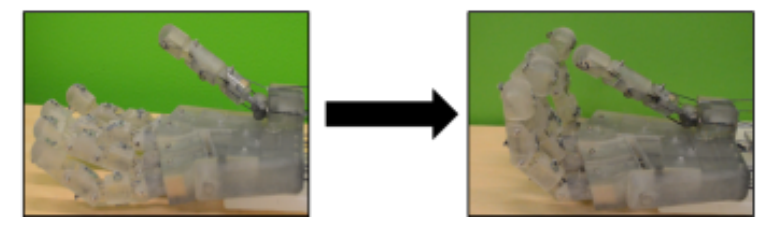

Fig. 1. Top: Data collection in 3 scenarios in unconstrained environment. Below: Using the dictionary of SEMs on the data collected, we find a temporal encoding of the behaviour by identifying at each moment in time the most relevant Eigenmotion. Joint velocities of the 5 digits. Below: "Behavioural barcode". Representations of a single Sparse Eigenmotion (SEM) producing the grasp indicated by the robotic hand. Figure taken and adapted from [1]. 


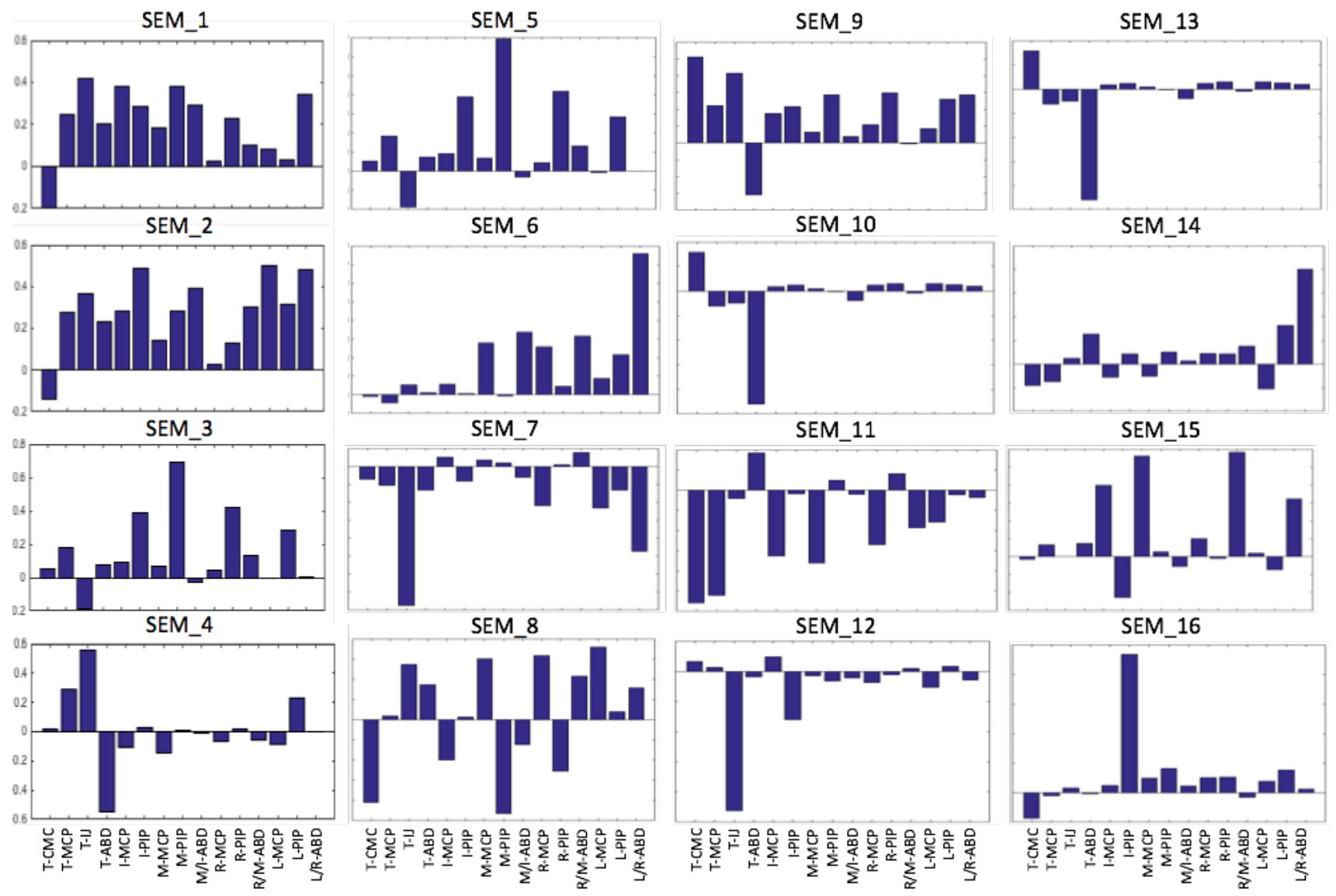

Fig. 2. Formal representation of 16 SEMs derived from our Sparse Coding algorithm. Bottom: Joints of the hand. Left: Bar height indicates the relative joint movement.

for different grasp types in the ventral premotor area F5 of the macaque cortex [19] and that the daily life correlation structure of finger joints predicts correlated activity between finger regions of primary motor cortex [20]. Indeed, our sparse code explained more of the variance in natural hand movements than correlation patterns or principle components as derived by classic PCA [1]. We set out here to explore how these results from human kinematics and computational neuroscience play out when we embody them in a robotic system - a novel dexterous robotic hand with a ball-jointed thumb [2].

The learnt dictionary consists of 45 SEMs and here we present 16 SEMs (see Figure 2). The bar height of the each sub-figure, represents the relative movement of each joint. SEMs were exported from the algorithm in a vector form. Each SEM vector contains joint velocity information, i.e. change in position information for 15 out 24 hand joints.

\section{RESULTS}

In order to test the effectiveness of our data-driven framework, we have applied the extracted SEMs on our highly dexterous robotic hand, the EthoHand [2]. The EthoHand designed in order to replicate the capabilities of the human hand for in-hand object manipulation (e.g. moving a ball between fingers or messaging on a smart phone), allowing us to map human hand kinematics to a naturalistic anthropomorphic hand. It carries $24 \mathrm{DoF}$ and it is under-actuated using 18 servo motors (HS-422, HighTech, Hitech RCD Inc., Poway, CA). The servo-motors were communicating with the computer via a micro-controller (Arduino Mega 2560, Arduino, Italy). Our novel articulation of the thumb our robotic hand allows for in-hand manipulation of complex objects in a naturalistic manner and thus to realistically implement the SEMs we measured from human data.

We designed a specialised Graphical User Interface (GUI) in MatLab that maps each joint velocity to the appropriate joint on the EthoHand, which also sends SEMs onto the hand's micro-controller. We implemented 4 SEMs on the robotic hand (see Figure 3) to demonstrate the ability of each SEM to actuate and manipulate all DoF of our dexterous robotic hand. Subsequently, we tested the physical capabilities of each SEM to statically hold objects of everyday life by performing effectively: large diameter grasp, power grasp with palm-arch joints activation, tripod, and precision grasp (see [21] for details). Hand control was entirely in openloop mode merely implementing the specific SEM, thus no specific grasp shaping, feedback on contact, joint or motor feedback information were used to implement the grasps. The hand was made of 3D printed, transparent ABS plastic, and was not coated with anti-slip surfaces. There, grasps 

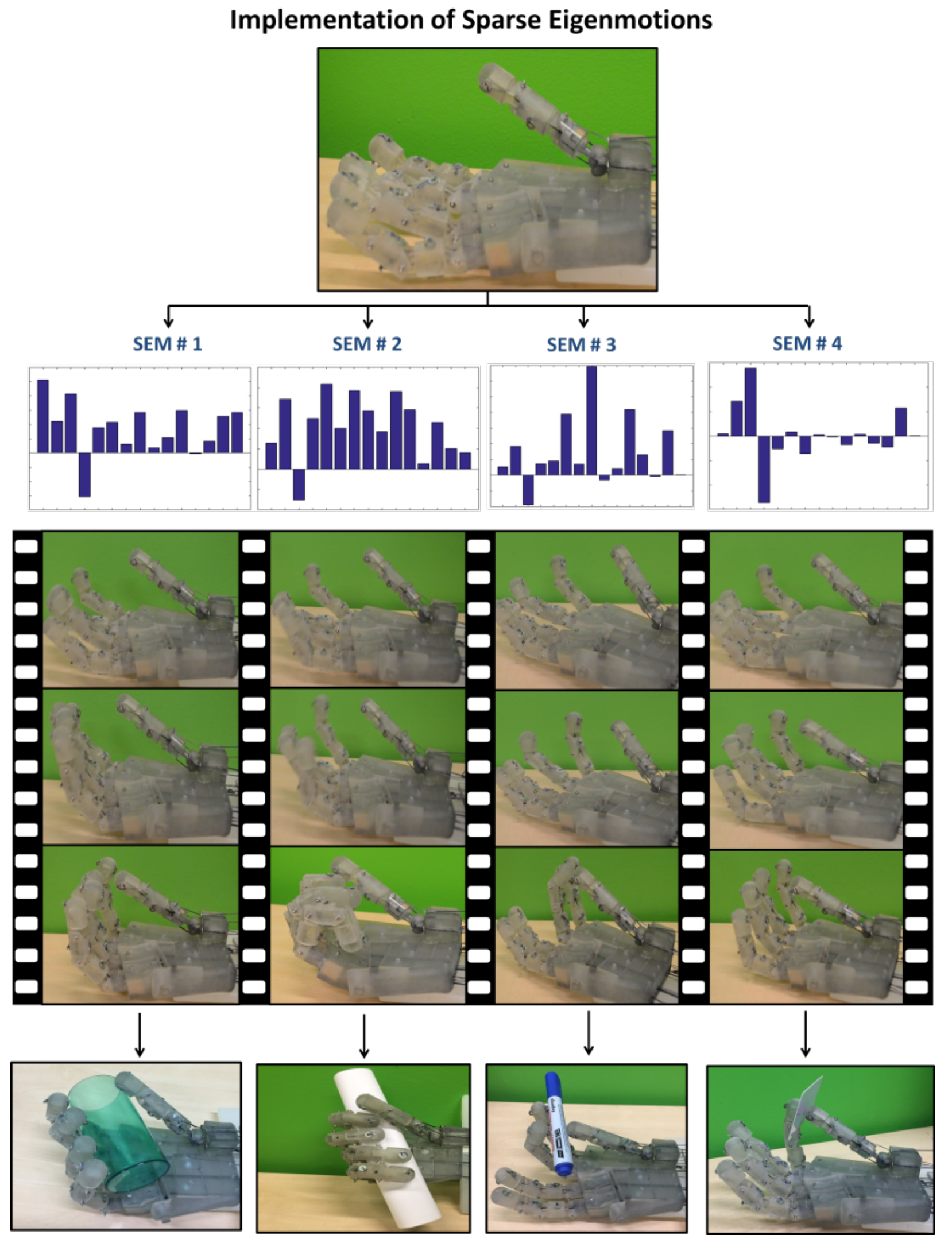

Fig. 3. Top: Our 3D printed dexterous artificial hand [2]. Below: SEMs loadings of each joint, Below: Implementation of the 4 most commonly used SEMs across our dataset, on a dexterous artificial hand, the EthoHand. We show 3 frames until final static posture in order to demonstrate the ability of SEMs to manipulate all DoF of our dexterous robot hand. Bottom: Subsequently we show that each SEM is capable of statically holding objects (See also attached video). 
were determined as stable if the objects placed into the the data, did not move or fall out of the hand within 60 seconds of holding.

\section{CONCLUSION}

Here we presented a novel approach towards the naturalistic control dexterous robotic hands by exploiting the statistics of natural movement. Data collected from unconstrained environments were analysed using the concept of sparse coding in order to derive the primitive hand motions across our dataset. We have previously shown that SEMs have a tremendous advantage over classic PCA to analyse hand motor control, as these form a sparse time-scale and amplitude invariant set of motion primitives[1]. For the same number of components, sparse codes explain a significantly larger amount of variance in natural hand movement data than PCA. Note, that in contrast to PCA type analysis the number of motion modes in SEM is not a function of the dimensionality of the data (PCA on D dimensional kinematics yields D principal components), but instead is a function of the actual structure of the data across time and dimensions (i.e. the number of SEMs can be higher or lower than the dimensionality of the kinematics). Subsequently, we implement the 4 commonly used SEMs across our data set, on our dexterous robotic hand. The static postures performed by our artificial hand indicate the ability of the SEMs to have a physical interpretation by performing stable grasping of daily life objects in open-loop control.

The capability of grasping and manipulating an object in a suitable, stable and control way is an outstanding feature for a robot, and thus far, one of the major problems to be solved in robotics [22]. This is due to the large amount of information a human can reasonably specify within a sufficiently small update interval is often far less a robot's DoF [23]. Yet, the very existence of stereotyped SEMs suggests that there are specific patterns in which these are used during daily life actions. If we wish to reproduce human-likeness in robotic end-effectors we should draw inspiration from the biomechanical structure of the human hand as well as the neural strategies deployed by the brain [24].

In the human hand, a single muscle does not always connect a single articular but rather has a unique connective architecture between muscles and articulations, such as the interconnection of a multi-tendon muscle with several articulations [25]. Hand muscle synergies have been studied extensively [4], [5] in order to investigate the dimensionality of hand control. [26] suggests that there is convincing evidence that muscle synergies are used, in order to alleviate the computational burden required by the brain in order to control all the DoF of the hand. However, while the idea of muscle synergies is appealing, there is no clear path on how to test these, nor how the approach can be used to implement these on robotic actuators. In contrast, the use of kinematically derived SEMs produces a sparse and concise representation of human hand motions that we ported straightforward to a dextrous robotic hand.
Despite the achievements in biomimetic robotic hand design and control [27], an appropriate control strategy for dextrous hands has not yet been realised in robotics or prosthetics. In the teleoperation and prosthetic domain complex mechanical hands are challenged in supporting many daily life tasks, because control strategies are not intuitive to learn and operate [28]. In prosthetics, this leads to an abandonment rate of $35 \%$ for users of dextrous robotic myoelectric hands within 6 months, with end-users preferring hook-like or rigid cosmetic hands[29]. Moreover, it is commonly assumed that sensory feedback is essential for stable grasping of objects in humans - motivating a major effort in neural engineering to provide sensory feedback [30] - as well as in tactile object sensing in robotics [31]. Yet, we demonstrate here that simple open-loop implementations of SEM derived control models results in stable grasps of daily life objects. Our findings suggest that the SEMs can be used to simplify the interaction of high-dimensional dextrous end-effectors utilising a lower dimensional control manifold defined by the SEM. We previously showed in neuroprosthetic hands that we can boost decoding accuracy of movement intention [11] if we apply the known spatio-temporal structure of kinematics [32-35].

We speculate that by using SEM based control modes we have a path to implement hand control strategies that reflect the way our brain represents hand controls. Using SEMs as building blocks of control, instead of individual joints or PCA derived components for controlling motion, could make learning to control prosthetic hands or teleoperated robotic hands considerably easier, as these naturally derived control modes may feel more intuitive to the user. Similarly, using SEMs for robotic learning, could provide a rich set of anthropomorphic control modes for machine learning systems to operate on. Thus, as in human vision sparse coding of natural scenes in brains is dual to Gabor wavelet analysis in computer vision, we suggest that sparse coding of natural motions in our brains is dual to the problem of an, yet unknown, robotic control strategy.

\section{REFERENCES}

[1] A. A. Thomik et al., "Towards sparse coding of natural movements for neuroprosthetics and brain-machine interfaces," in IEEE Neural Engineering (NER), vol. 7, 2015, pp. 938-941.

[2] C. Konnaris et al., "Ethohand: A dexterous robotic hand with ball-joint thumb enables complex in-hand object manipulation," in IEEE Biorob, IEEE, vol. 6, 2016.

[3] R. Vinjamuri, M. Sun, et al., "Dimensionality reduction in control and coordination of the human hand," IEEE Trans on Biomed Eng, vol. 57, no. 2, pp. 284-295, 2010.

[4] M. Santello et al., "Postural hand synergies for tool use," J Neurosci, vol. 18, no. 23, pp. $10105-10115$, 1998. 
[5] A. D'Avella et al., "Combinations of muscle synergies in the construction of a natural motor behavior," Nat Neurosci, vol. 6, no. 3, pp. 300-308, 2003.

[6] V. C. K. Cheung et al., "Central and sensory contributions to the activation and organization of muscle synergies during natural motor behaviors.," Journal of Neuroscience, vol. 25, no. 27, pp. 6419-6434, 2005.

[7] M. C. Tresch and A. Jarc, "The case for and against muscle synergies.," Current Opinion in Neurobiology, vol. 19, no. 6, pp. 601-607, 2009.

[8] A. Bicchi et al., "Modelling natural and artificial hands with synergies.," eng, Philosophical transactions of the Royal Society of London. Series B, Biological sciences, vol. 366, no. 1581, pp. 3153-3161, 2011.

[9] M. Santello et al., "Hand synergies: Integration of robotics and neuroscience for understanding the control of biological and artificial hands," Physics of Life Reviews, pp. -, 2016.

[10] J. N. Ingram, K. P. Körding, I. S. Howard, and D. M. Wolpert, "The statistics of natural hand movements," Experimental Brain Research, vol. 188, no. 2, pp. 223-236, 2008.

[11] J. J. Belić and A. A. Faisal, "Decoding of human hand actions to handle missing limbs in neuroprosthetics," Frontiers in computational neuroscience, vol. 9, 2015.

[12] "Encyclopedia of neuroscience," in, M. D. Binder, N. Hirokawa, and U. Windhorst, Eds. Berlin, Heidelberg: Springer Berlin Heidelberg, 2009, ch. Efficient Coding Hypothesis, pp. 1037-1037.

[13] B. A. Olshausen and D. J. Field, "Sparse coding with an overcomplete basis set: a strategy employed by V1?"Vision research, vol. 37, no. 23, pp. 3311-3325, 1997.

[14] C. Gavriel and A. A. Faisal, "Wireless kinematic body sensor network for low-cost neurotechnology applications in-the-wild," in NER, 2013 6th Int IEEE/EMBS Conf, IEEE, 2013, pp. 1279-1282.

[15] R. MacCoun, J Pol Anal Manag, vol. 22, no. 2, pp. 330-332, 2003.

[16] W. Abbott et al., "Embodied salience for gaze analysis in ecologically valid environments.," $J$ of Vis, vol. 15, no. 12, pp. 366-366, 2015.

[17] B. A. Olshausen and D. J. Field, "Emergence of simple-cell receptive field properties by learning a sparse code for natural images," Nature, vol. 381, no. 6583, pp. 607-609, 1996.

[18] G Rizzolatti et al., "The organization of the cortical motor system: New concepts," Electroencephalography and Clinical Neurophysiology, vol. 106, no. 4, pp. $283-296,1998$.

[19] V. Raos et al., "Functional properties of graspingrelated neurons in the ventral premotor area F5 of the macaque monkey.," eng, Journal of neurophysiology, vol. 95, no. 2, pp. 709-729, 2006.

[20] N. Ejaz et al., "Hand use predicts the structure of representations in sensorimotor cortex.," Nature neuroscience, vol. 18, no. 7, pp. 1034-1040, 2015.
[21] T. Feix, et al., "The grasp taxonomy of human grasp types," IEEE Trans Hum Mach Syst, vol. 46, no. 1, pp. 66-77, 2016.

[22] L. Ascari et al., "Bio-inspired grasp control in a robotic hand with massive sensorial input," Biol $\mathrm{Cy}$ ber, vol. 100, no. 2, pp. 109-128, 2008.

[23] A. Tsoli et al., "2d subspaces for user-driven robot grasping," Robotics, Science and Systems Conference: Workshop on Robot Manipulation, vol. 2, pp. 7-2, 2007.

[24] M. Ciocarlie et al., "Dimensionality reduction for hand-independent dexterous robotic grasping," in IROS, 2007. IEEE/RSJ Int Conf, ID: 1, 2007, pp. 3270-3275.

[25] M.-J. Liu et al., "Biomechanical characteristics of hand coordination in grasping activities of daily living," PLoS ONE, vol. 11, pp. 1-16, Jan. 2016.

[26] E. Bizzi et al., "The neural origin of muscle synergies," Front Comput Neurosci, vol. 7, no. 51, 2013.

[27] J. T. Belter and A. M. Dollar, "Performance characteristics of anthropomorphic prosthetic hands," in IEEE ICORR, 2011, pp. 1-7.

[28] J. Ayers et al., Neurotechnology for biomimetic robots. MIT press, 2002.

[29] E. A. Biddiss and T. T. Chau, "Upper limb prosthesis use and abandonment: A survey of the last 25 years," Prosth and Orth Int, vol. 31, no. 3, pp. 236-257, 2007.

[30] S. J. Bensmaia and L. E. Miller, "Restoring sensorimotor function through intracortical interfaces: Progress and looming challenges," Nature Reviews Neuroscience, vol. 15, no. 5, pp. 313-325, 2014.

[31] F. M. P. Behbahani, G. Singla?Buxarrais, and A. Faisal, "Haptic slam: An ideal observer model for bayesian inference of object shape and hand pose from contact dynamics," in $F, 2015$.

[32] A. A. C. Thomik et al., "Real-time movement prediction for improved control of neuroprosthetic devices," in IEEE Neural Engineering (NER), 2013, pp. 625628.

[33] D. Haber et al., "Unsupervised time series segmentation for high-dimensional body sensor network data streams," in Wearable and Implantable Body Sensor Networks (BSN), 2014 11th International Conference on, 2014, pp. 121-126.

[34] M. Xiloyannis, C. Gavriel, A. A. Thomik, and A. A. Faisa, "Gaussian process regression for accurate prediction of prosthetic limb movements from the natural kinematics of intact limbs," in IEEE Neural Engineering (NER), IEEE, 2015, pp. 659-662.

[35] M. Xiloyannis, C. Gavriel, A. A. Thomik, and A. A. Faisal, "Dynamic forward prediction for prosthetic hand control by integration of emg, mmg and kinematic signals," in IEEE Neural Engineering (NER), IEEE, 2015, pp. 611-614. 